\title{
SIMULADOR DE PROCESSO E ALARMES DO SISTEMA DE CARREGAMENTO DO ALTO FORNO 2*
}

\author{
Moacir Alves dos Reis ${ }^{1}$ \\ Roberto de Oliveira Mariano² \\ Ronaldo Antônio Mendes ${ }^{3}$
}

\section{Resumo}

O objetivo deste trabalho foi criar um projeto de um simulador do sistema de carregamento do Alto Forno 2, voltado para o treinamento de operadores e técnicos em manutenção. O principal objetivo está em produzir uma ferramenta que auxilie no processo de ensino dos operadores e técnicos de forma mais rápida e segura, reduzindo os custos do treinamento e ao mesmo tempo simulando situações de operação, alarmes de processos sem interferência no sistema de carregamento real. São apresentadas as etapas previstas para o desenvolvimento do projeto e as ações já realizadas até o presente momento. O simulador será equipado com equipamentos reais do sistema de carregamento do Alto Forno, sendo apenas simulado as entradas e saídas físicas $(\mathrm{I} / \mathrm{O})$ dos equipamentos externos (motores, limites, válvulas, etc.) e utilizará um ambiente virtual modelado a partir do PLC, SDCD e Monitores para que os operadores possam visualizar e simular todos os alarmes deste processo.

Palavras-chave: Simulador; Alto Forno; PLC/SDCD.

\section{PROCESS SIMULATION AND ALARMS SYSTEM CHARGING BLAST FURNACE 2}

\section{Abstract}

The objective of this work was to create a design of a simulator charging Blast Furnace 2 system, focusing on the training of operators and maintenance technicians. The main objective is to produce a tool that assists in the process of the operators and technicians training in a quicker and more secure way, reducing training costs while simulating operating situations, alarms processes without interfering with the actual charging system. Steps planned for the project development and the actions already taken to date are presented. The simulator will be equipped with real equipment of the blast furnace charging system and only simulated the physical inputs and outputs $(\mathrm{I} / \mathrm{O})$ of the external equipment (engines, limits, valves, etc.) And use a virtual environment modeled after the PLC, DCS and monitors to enable operators to visualize and simulate all alarms of this process.

Keywords: Simulator; Blast Furnace; PLC/SDCD.

Senior Engineer, CSN, Brasil.

Technical specialist, CSN, Brasil.

Technical Specialist, CSN, Brasil.

* Contribuição técnica ao $18^{\circ}$ Seminário de Automação e TI Industrial, 23 a 26 de setembro de 2014, São Paulo, SP, Brasil. 


\section{INTRODUÇÃO}

Com o atual desenvolvimento tecnológico na Siderurgia, a automação de todos os equipamentos se fez necessário para aumentar a produtividade, segurança, redução de custos, etc. Observamos também que um sistema de automação bem elaborado faz com que os equipamentos trabalhem sempre em automático, sem a interferência dos operadores. Porém com este avanço da tecnologia, surgem também as seguintes

dúvidas:

1) Quando ocorrer algum problema nos equipamentos, a operação e manutenção conseguirão resolver os problemas o mais rápido possível?

2) O operador terá habilidade suficiente para operar os equipamentos em manual? Sabemos que o sistema está sempre funcionando em automático.

Optamos por criar a Realidade Virtual no processo de Carregamento e Topo do Alto Forno 2. Neste trabalho será possível visualizar todo o processo de simulação de operação e falhas de todos os equipamentos (bombas, válvulas, motores, etc.) auxiliando assim o aprendizado da operação e manutenção na resolução de problemas e operação dos equipamentos em condição manual em condições reais. Conseguimos também reduzir os riscos de alteração no processo de nosso Alto Forno, pois a criação da lógica, telas do Scada e I/Os serão todos desenvolvidos e testados no sistema de Simulação.

\section{SISTEMA DE CARREGAMENTO E TOPO DO ALTO FORNO 2}

O sistema de carregamento do Alto Forno que foi simulado é composto de silos de estocagem, alimentadores, peneiradores, balanças e correias.

Todas as matérias primas são armazenadas nos silos de estocagem e pesadas nas balanças de acordo com o leito de fusão programado pelo operador na matriz de carregamento. Após o pedido de carga, a balança programada descarrega todo o material na correia M2 que alimentará o Topo do Forno.

Os equipamentos do Topo são utilizados para o carregamento das matérias primas no interior do Alto Forno. Os equipamentos do Topo são constituídos de balanças, válvulas, calha distribuidora e sistema hidráulico.

Para monitorar o nível de material no interior do Alto Forno são utilizadas sondas de nível radar e eletromecânica. Esta medição é utilizada para iniciar o carregamento do Alto Forno através da balança e calha distribuidora. Neste momento também é liberado nova descarga das balanças da Stock House para completar a balança de Topo que será descarregada. As figuras 1, 2 e 3 ilustram este processo.

* Contribuição técnica ao $18^{\circ}$ Seminário de Automação e TI Industrial, 23 a 26 de setembro de 2014, São Paulo, SP, Brasil. 
AUTOMAÇÃO \& TI

$18^{\circ}$ SEMINARIO DE

AUTOMAÇÃO \& TI INDUSTRIAL
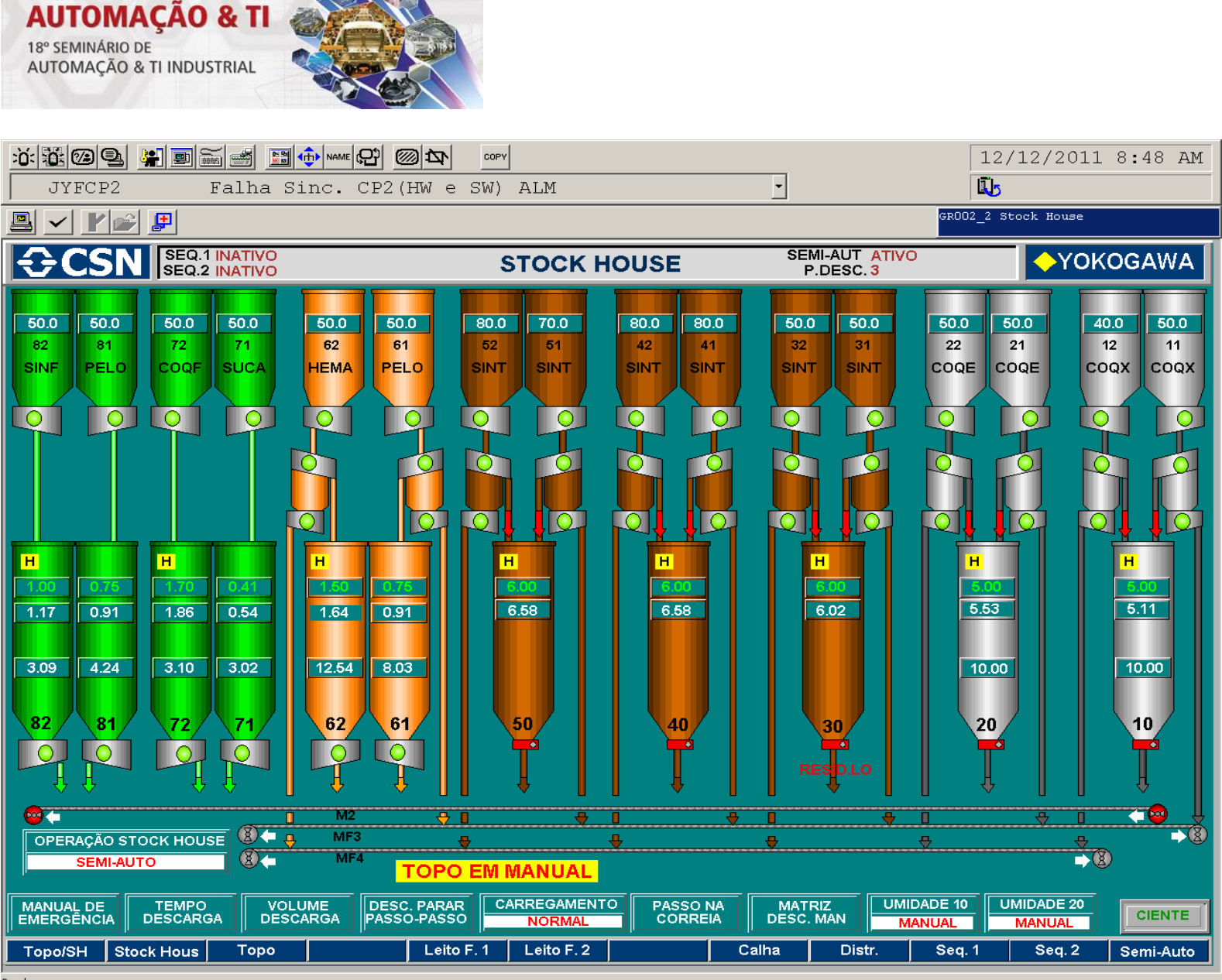

Figura 1 - Processo de armazenagem e pesagem das matérias-primas

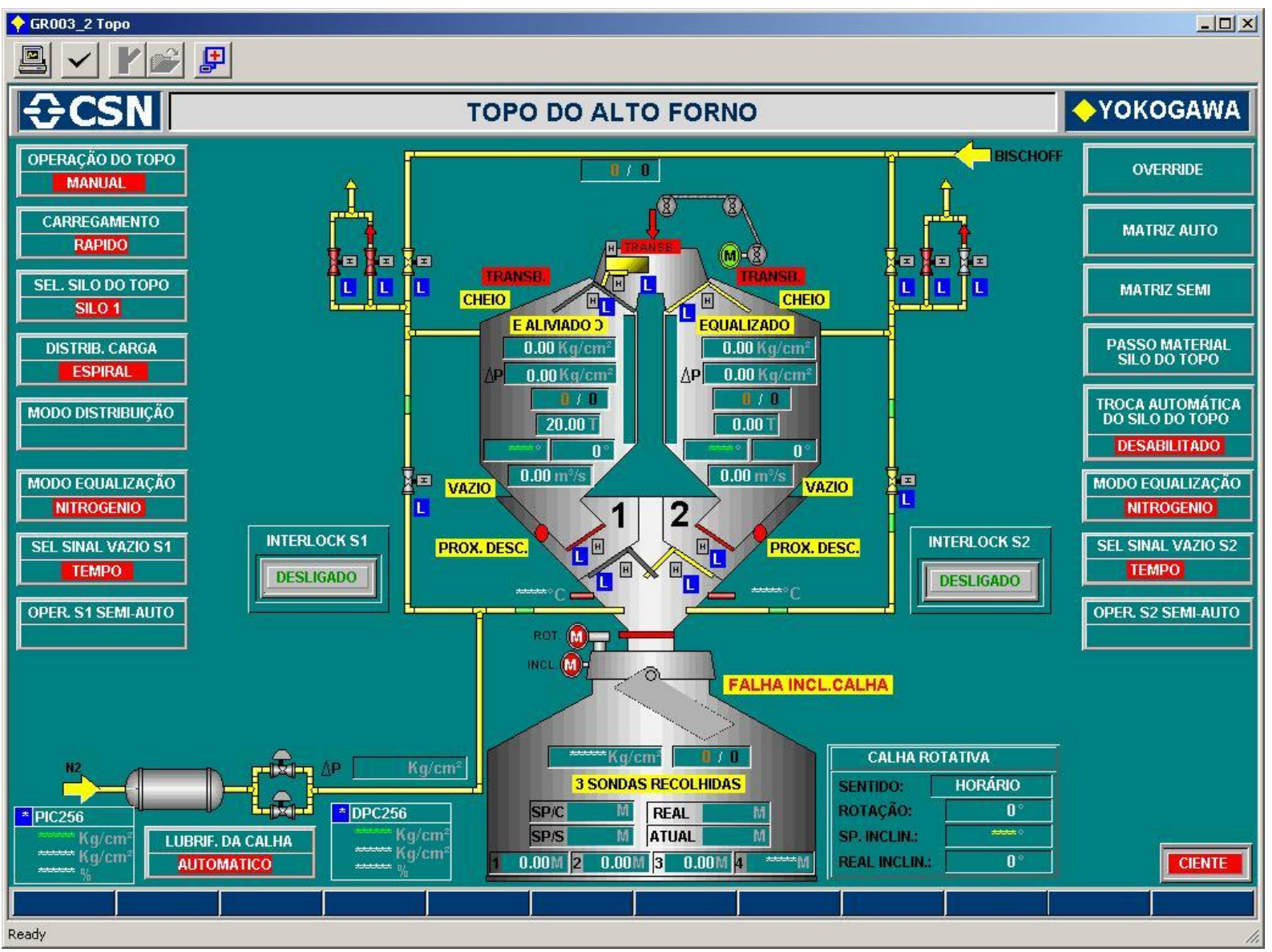

Figura 2 - Processo de carregamento do Alto Forno

* Contribuição técnica ao $18^{\circ}$ Seminário de Automação e TI Industrial, 23 a 26 de setembro de 2014, São Paulo, SP, Brasil. 

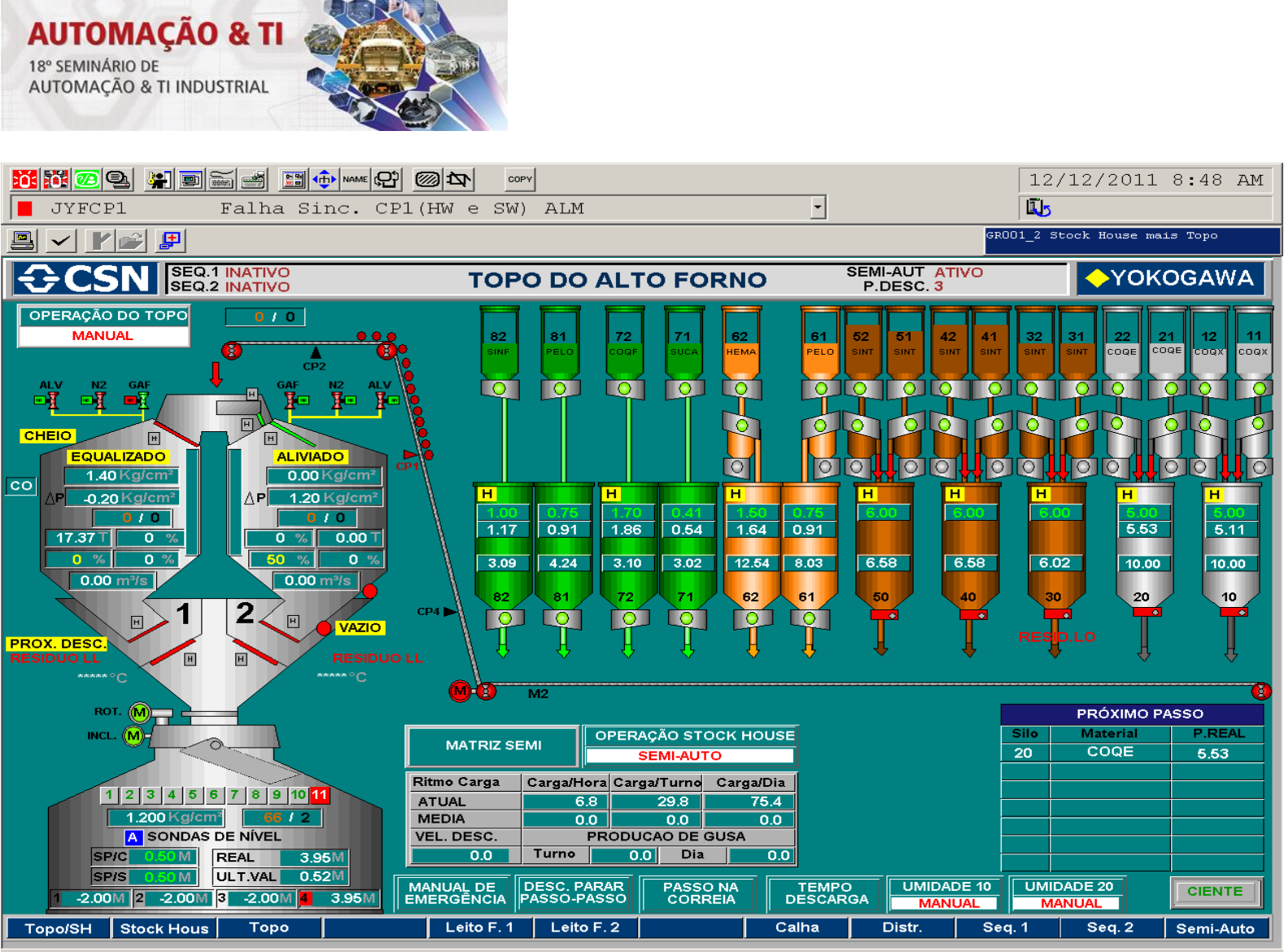

Figura 3 - Processo completo de carregamento do Alto Forno

\section{SISTEMA DE AUTOMAÇÃO DO ALTO FORNO 2}

O sistema de automação do Topo e Stock House é composto de um SDCD da Yokogawa e um PLC da Rockwell [1-2]. O sistema anterior era obsoleto e utilizava ainda o painel sinótico, dificultando a vida do operador para operar e identificar as falhas ocorridas no sistema de carregamento. Foi desenvolvido na Yokogawa em parceria com a CSN o novo sistema de automação para este processo. Observamos também que com este novo sistema, o operador poderia ter dificuldades durante a operação deste sistema, apesar de todos receberem o treinamento tradicional. A figura 4 ilustra o novo sistema de automação do Alto Forno.

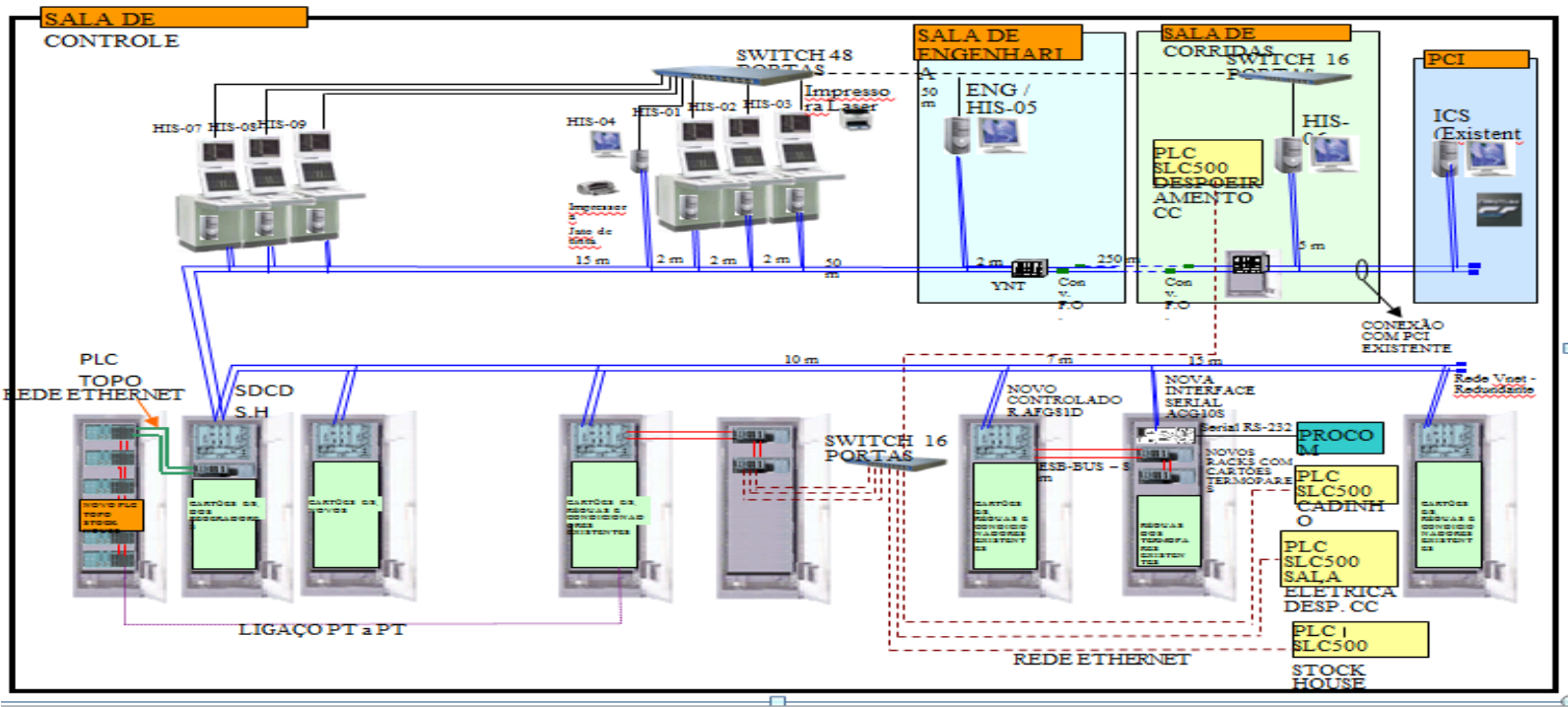

Figura 4 - Sistema de automação do Alto Forno

* Contribuição técnica ao $18^{\circ}$ Seminário de Automação e TI Industrial, 23 a 26 de setembro de 2014, São Paulo, SP, Brasil. 


\section{PROJETO DO SIMULADOR DO CARREGAMENTO DO ALTO FORNO}

\subsection{Tomada de Decisão}

- Implantamos uma tecnologia nova para o carregamento do Alto Forno;

- Operadores sempre relataram muita dificuldade no aprendizado deste processo, devido ao sistema sempre estar operando em automático;

- Falta de habilidade do operador para operar o carregamento do Alto Forno em manual;

- Os operadores trabalham em regime de turno de revezamento, dificultando o treinamento coletivo;

- Dificuldade para identificar e solucionar os alarmes que ocorriam durante o processo.

\subsection{Objetivos}

- Produzir uma ferramenta que auxilie no processo de ensino a manutenção elétrica e instrumentação de forma mais rápida e segura;

- Reduzir os custos do treinamento e o tempo de permanência do aprendiz no Sistema real;

- Melhorar a habilidade do operador para trabalhar com o carregamento em manual;

- Reduzir/eliminar perda de produção;

- Solucionar os problemas rapidamente;

- Desenvolver e testar melhorias no processo do Alto Forno.

- Aumentar a eficiência e redução de custos relacionados a equipamentos e ao tempo de desenvolvimento de novas aplicações;

- Desenvolver novas tecnologias para serem utilizadas neste tipo de projeto;

- Aumentar o moral dos operadores;

\subsection{Solução Adotada}

- Criar um simulador do Sistema de carregamento na plataforma do projeto novo do Alto forno 2, porém o sistema trabalha independente do processo principal, evitando interferências no mesmo;

- Desenvolver software de dispositivos de force feedback, simulando limites, motores, comportas, válvulas, sensores e alarmes;

- Desenvolver o software utilizando o mesmo projeto instalado no PLC e SDCD.

\subsection{Funcionalidades com este Simulador}

- Pode ser testada toda a lógica do sistema utilizado no software da Stock House e Topo, eliminando assim erros na programação;

- Desenvolver melhorias no sistema virtual e verificar efeitos colaterais antes de inserir nas CPUs que operam o Alto Forno;

* Contribuição técnica ao $18^{\circ}$ Seminário de Automação e TI Industrial, 23 a 26 de setembro de 2014, São Paulo, SP, Brasil. 


\subsection{Arquitetura do Sistema de Automação do Simulador do Alto Forno 2}

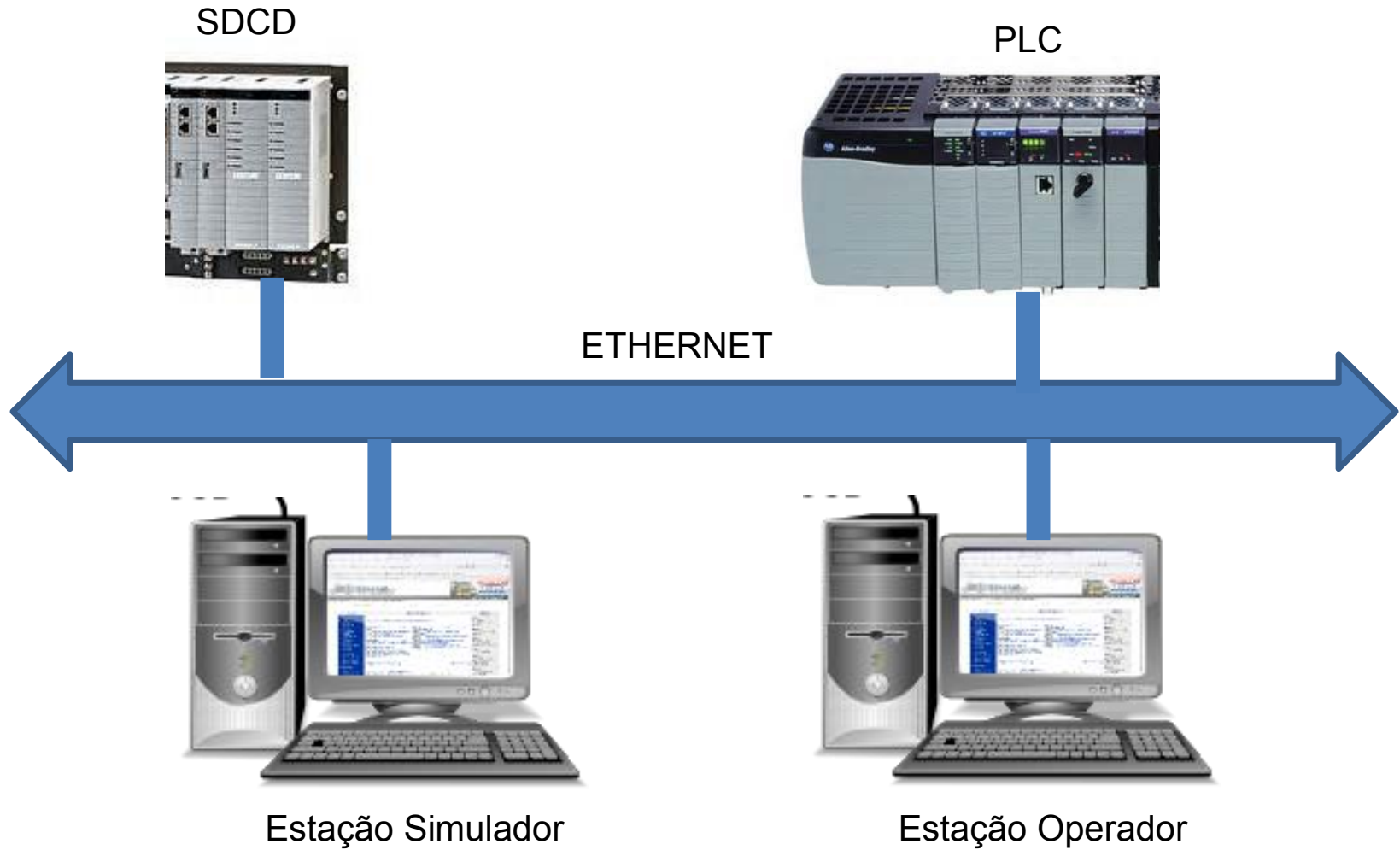

\subsection{Arquitetura das telas do Simulador}

- Todas as telas que o operador visualiza, são as mesmas telas que estão operando no projeto principal;

- Foram criadas telas auxiliares para o operador testar todos os alarmes existentes no sistema de carregamento do Alto Forno;

- Todo o sistema de carregamento do simulador opera da mesma maneira que o sistema principal, ou seja, o operador pode testar qualquer tipo de carregamento antes de aplicar no sistema principal.

\subsection{Telas auxiliares para a Simulação de Alarmes}

Foram desenvolvidas 10 telas para a simulação dos alarmes na Stock House e Topo do Alto Forno. Mostraremos abaixo, através das figuras 5, 6 duas telas que são utilizadas pelo operador durante a simulação dos alarmes. As telas de operação do Alto Forno continuam as mesmas utilizadas no sistema principal.

* Contribuição técnica ao $18^{\circ}$ Seminário de Automação e TI Industrial, 23 a 26 de setembro de 2014, São Paulo, SP, Brasil. 


\section{BALANGA 910}

\begin{tabular}{|l|l|l|}
\hline ID & EVT - DESCARGA (910) & PV \\
\hline 01 & FI. Limite de Aberto & \\
\hline 02 & FI. Limite de Fechado & \\
\hline 03 & FI. Ret. PI Abrir (CCM) & \\
\hline 04 & FI. Ret. pl Fechar (CCM) & \\
\hline 05 & Lim. Abertoffech Atuados & \\
\hline 06 & Sobrecarga na Comporta & \\
\hline 07 & Residuo HH & \\
\hline 08 & Residuo HI & \\
\hline 09 & Residuo LO & \\
\hline 10 & Residuo LL \\
\hline 11 & IOP - da Balanca \\
\hline 12 & E Reserva & \\
\hline 13 & Reset Tara Manutençao & \\
\hline 14 & Sonda Transb. da Balanca & \\
\hline 15 & Chave Local da Comporta & \\
\hline
\end{tabular}

FLUXO CARGA I DESCARGA

0. 280

Chave Local do Vibr.

FI. Retorno Liga Pen.

FI. Retorno Desliga Pen.

Sobrecarga no Pen.

Chave Local do Pen.

Sil.Finos Sonda Niv. Max.

Nivel Baixo no Silo Estoc.

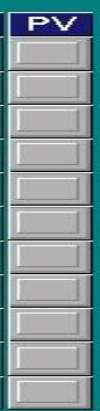

ID EVT - CARREG. (912)

01 FI. Retorno Liga Vibr.

02 FI. Retorno Desliga Vibr.

03 Sobrecarga no Vibr.

04 Chave Local do Vibr.

05 FI. Retorno Liga Pen.

06 FI. Retorno Desliga Pen.

07 Sobrecarga no Pen.

08 Chave Local do Pen.

09 Sil.Finos Sonda Nív. Max.

PARADA DE EMERGENCIA S.H.

Botoeira de Campo JYF909

Figura 5 - Tela de simulação de alarmes da Stock House

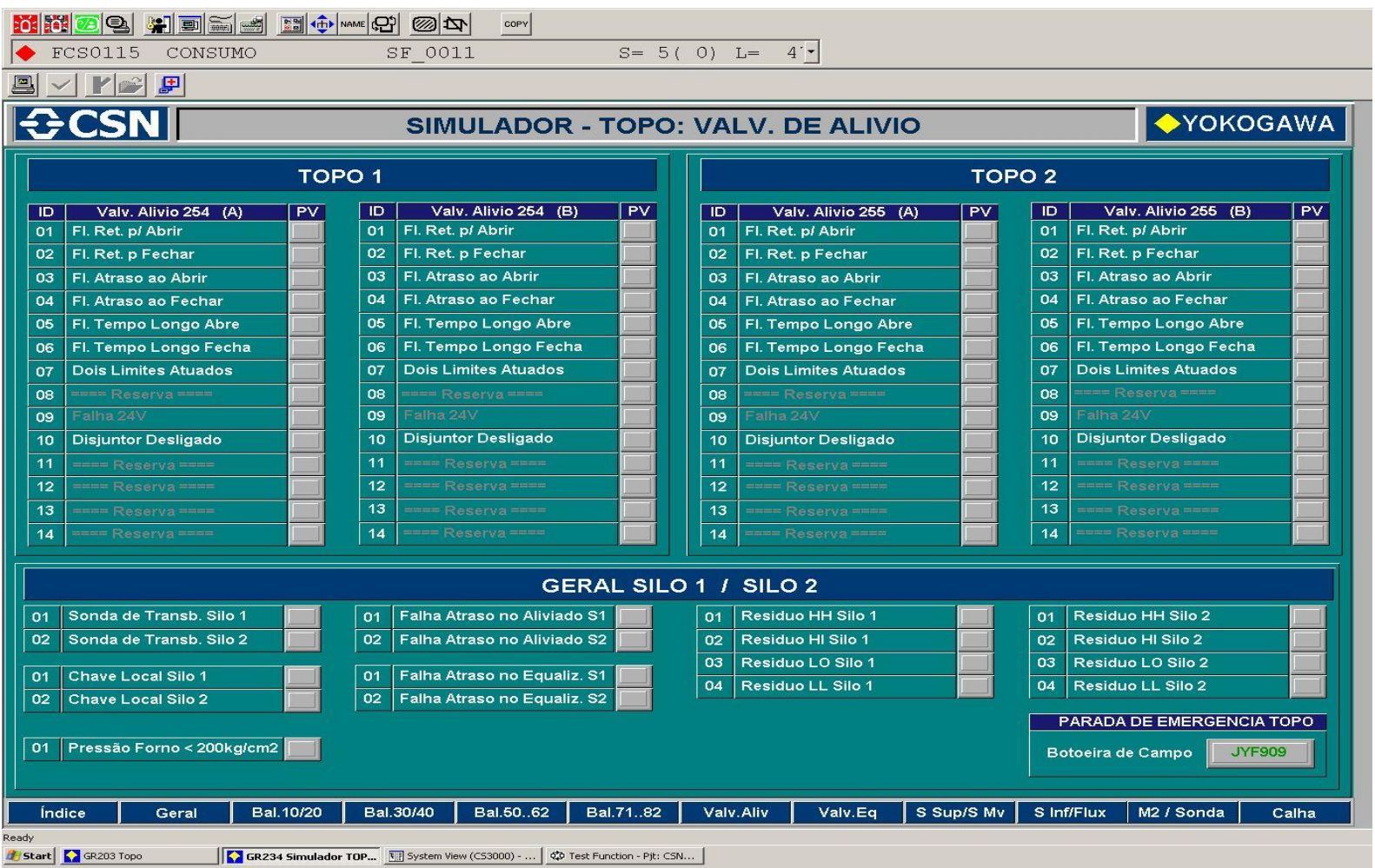

Figura 6 - Tela de simulação de alarmes do Topo

\section{PRINCIPAIS RESULTADOS OBTIDOS}

- Elaboração do manual para a utilização do simulador de alarmes e processo, conforme mostrado na figura 7 e 8;

* Contribuição técnica ao $18^{\circ}$ Seminário de Automação e TI Industrial, 23 a 26 de setembro de 2014, São Paulo, SP, Brasil. 
- Elaboração do guia de solução para os alarmes do processo do carregamento, conforme mostrado na figura 9 e 10;

- Criação do Centro de Treinamento para os atuais e novos operadores, conforme mostrado na figura 11;

- Redução no tempo de solução dos problemas nos equipamentos;

- Operação padronizada dos operadores do carregamento.
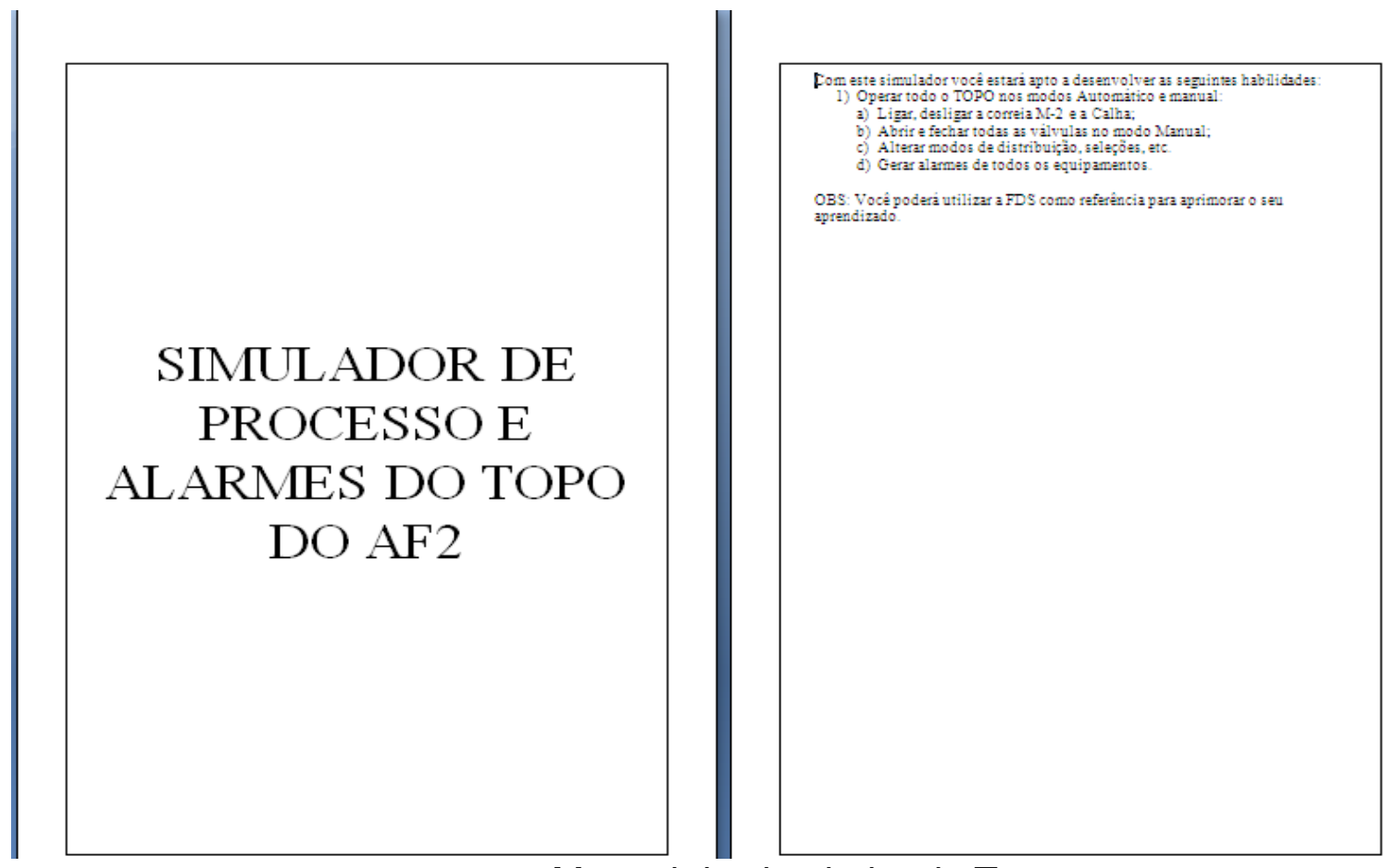

Figura 7 - Manual do simulador do Topo

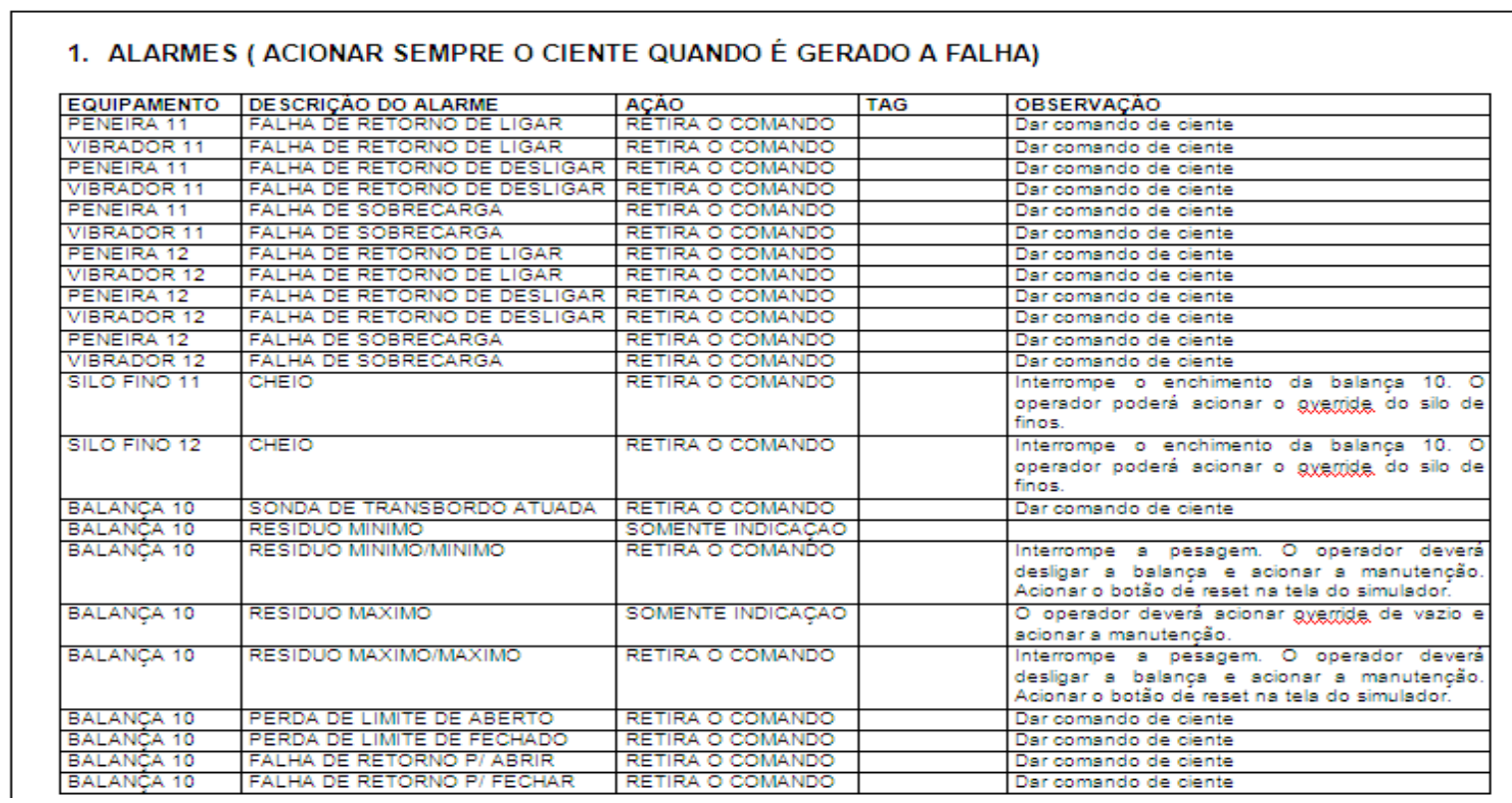

Figura 8 - Manual de alarmes do carregamento

* Contribuição técnica ao $18^{\circ}$ Seminário de Automação e TI Industrial, 23 a 26 de setembro de 2014, São Paulo, SP, Brasil. 


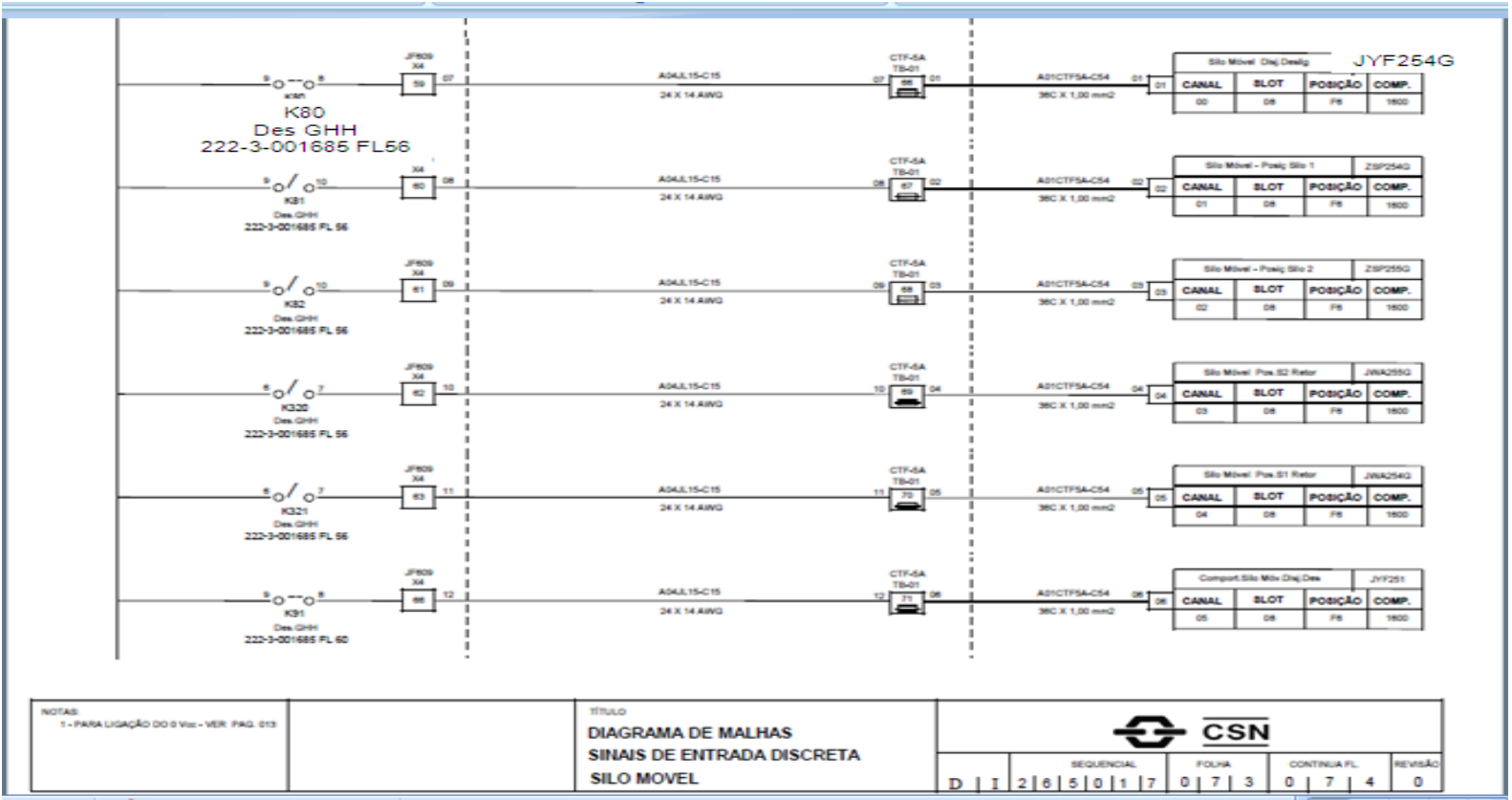

Figura 9 - Diagrama de malhas com o tag da falha ocorrida

\begin{tabular}{|c|c|c|c|c|c|}
\hline Tag. & Alarme & Normal & $\begin{array}{l}\text { Condição } \\
\text { de Alarme }\end{array}$ & Causas Possiveis & $\begin{array}{c}\text { Ação } \\
\text { Operador/manutenção }\end{array}$ \\
\hline JWTLA254G & $\begin{array}{l}\text { Tempo Longo do Silo } \\
\text { Móvel Para o Silo } 1 \text { do } \\
\text { topo. }\end{array}$ & Normal & Falha & $\begin{array}{ll}1 . & \text { Falha no comando para } \\
\text { mover o silo móvel. } \\
\text { malar } \\
\text { Falha no retorno para } \\
\text { mover o silo movel para o } \\
\text { silo } 1 \text { ou } 2 . \\
\text { 3. Falha do limite silo movel } \\
\text { no silo } 1 \text { ou silo } 2 . \\
\text { 4. Falha no sistema } \\
\text { hidráulico. }\end{array}$ & $\begin{array}{l}\text { 1. Verificar entrada, no } \\
\text { PLC e painel } \\
\text { CTE(conforme } \\
\text { diagrama de malha do } \\
\text { topo) } \\
\mathbf{N}^{\circ} \text { DI265017 / FL73 }\end{array}$ \\
\hline JWTLF255G & $\begin{array}{l}\text { Tempo Longo do Silo } \\
\text { Móvel Para o Silo } 2 \text { do } \\
\text { topo. }\end{array}$ & Normal & Falha & 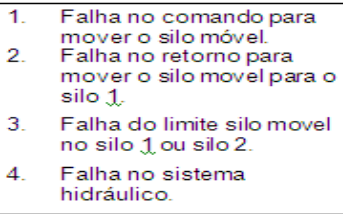 & $\begin{array}{l}\text { 1. Verificar entrada, no } \\
\text { PLC e painel } \\
\text { CTF(conforme } \\
\text { diagrama de malha do } \\
\text { topor) } \\
\mathbf{N}^{\circ} \text { DI265017 / FL73 }\end{array}$ \\
\hline $\begin{array}{l}\text { JWA154G } \\
\text { JWA255G }\end{array}$ & $\begin{array}{l}\text { Falha de Retorno silo } \\
\text { movel para Silo } 1 \text { ou } \\
2 .\end{array}$ & Normal & Falha & $\begin{array}{l}\text { Falha do relé, no CCM, ou } \\
\text { entrada do PLC. }\end{array}$ & $\begin{array}{l}\text { 1. Verificar,entrada, no } \\
\text { PLC e painel } \\
\text { CTF(conforme } \\
\text { diagrama de malha do } \\
\text { topo) } \\
\mathbf{N}^{\circ} \text { DI265017 / FL73 }\end{array}$ \\
\hline JYF254G & $\begin{array}{l}\text { Disjuntor Desligado } \\
\text { (silo movel do topo). }\end{array}$ & Normal & Falha & 1. Falha de energia ou local. & $\begin{array}{l}\text { 1. Verificar entrada, no } \\
\text { PLC e painel } \\
\text { CTE(conforme } \\
\text { diagrama de malha do } \\
\text { topo) } \\
\mathbf{N}^{\circ} \text { DI265017 / FL73 }\end{array}$ \\
\hline
\end{tabular}

Figura 10 - Guia para solucionar os alarmes do diagrama de malhas 

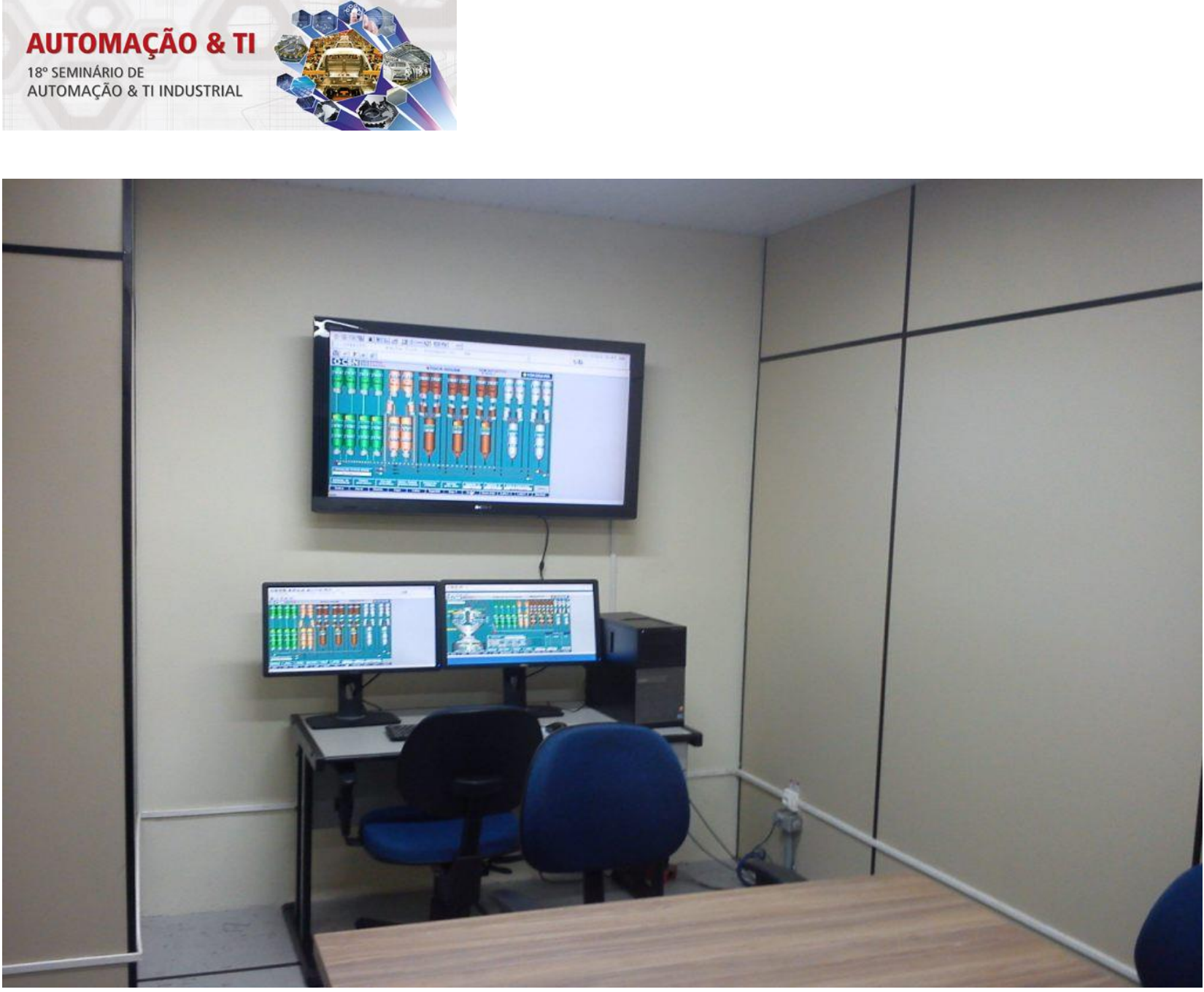

Figura 11 - Centro de Treinamento do Carregamento do Alto Forno

\section{CONCLUSÃO}

Com o uso deste Simulador foi possível testar todo o carregamento de forma muito próxima das condições reais, antecipando correção de problemas muito antes da partida deste novo sistema. Todos os operadores foram treinados por este novo método de ensino. Tivemos excelentes resultados na redução de problemas devido a falta de habilidade para operar o Alto Forno em manual, ganhamos também com a solução mais rápida dos alarmes. Implantamos o Centro de Treinamento para todos os colaboradores novos e atuais para o processo de reciclagem do carregamento do Alto Forno.

Devido ao grande sucesso deste projeto, foi iniciado e concluído também o Simulador de todo o processo do Alto Forno 3 (Carregamento, Regeneradores, Limpeza de gás, Sala de Corridas e Monitoração).

\section{REFERÊNCIAS}

1 Manual de programação RSLOGIX 5000 da Rockweel Automation;

2 Manual de programação do SDCD Centum CS3000 da Yokogawa.

* Contribuição técnica ao $18^{\circ}$ Seminário de Automação e TI Industrial, 23 a 26 de setembro de 2014, São Paulo, SP, Brasil. 